\title{
大别山黄镇榴辉岩锆石的微区微量元素分析: 榴辉岩相变质锆石的微量元素特征
}

\author{
吴元保 ${ }^{\mathbb{1}}$ 陈道公 ${ }^{\mathbb{1} *}$ 夏群科 ${ }^{\mathbb{1}}$ 涂湘林 ${ }^{(2)}$ 程 吴 ${ }^{\mathbb{1}}$ \\ (1)中国科学技术大学地球与空间科学系, 合肥 230026; (2)中国科学院广州地球化学研究所, 广州 510640. \\ *联系人, E-mail: dgchen@ustc.edu.cn)
}

\begin{abstract}
摘要对大别山黄镇朱家冲榴辉岩锆石进行阴极发光显微结构分析, 确定出核部原岩岩浆锆石和边部 的变质增生锆石。在此基础上利用激光探针等离子体质谱对不同区域进行了微量元素测定，结果表明 原岩锆石和变质增生锆石具有不同的微量元素组成. 原岩锆石的微量元素含量高且变化范围大; 变质 锆石的重稀土元素含量低(132.2 197.6 $\mu \mathrm{g} / \mathrm{g})$, 重稀土的分异程度小 $\left((\mathrm{Yb} / \mathrm{Gd})_{\mathrm{CN}}=8.6 \sim 11.9\right)$, 说明变质 锆石与富集重稀土的石榴石平衡结晶. 边部锆石的 $\mathrm{Nb}, \mathrm{Ta}$ 含量和 $\mathrm{Nb} / \mathrm{Ta}$ 值分别为 $0.5 \sim 1.4 \mu \mathrm{g} / \mathrm{g}, 0.7 \sim$ $1.5 \mu \mathrm{g} / \mathrm{g}$ 和 $0.3 \sim 1.3$, 明显低于核部 ( $\mathrm{Nb}$ 和 $\mathrm{Ta}$ 的含量分别为 $3.8 \sim 19.7$ 和 $2.7 \sim 12.1 \mu \mathrm{g} / \mathrm{g}, \mathrm{Nb} / \mathrm{Ta}$ 值为 1.0 4.6). 这一结果证明边部的变质锆石 $\mathrm{Nb}, \mathrm{Ta}$ 特征是与榴辉岩中的峰期变质矿物金红石平衡结晶的结果. 锆石 的稀土元素配分模式和 $\mathrm{Nb}, \mathrm{Ta}$ 等微量元素特征表明, 边部的变质锆石形成于榴辉岩相变质作用期间. 因此, 锆石的微量元素分析可以为锆石的形成环境提供新的制约.
\end{abstract}

\section{关键词 榴辉岩 锆石 微量元素 金红石}

锆石是各类岩石中广泛存在的副矿物. 由于锆 石富 $\mathrm{U}$, 低普通 $\mathrm{Pb}$ 含量, 使其成为精确 U-Pb 计时的 首选对象. 但对于复杂的变质岩而言, 锆石 U-Pb 年 龄的合理解释还存在困难. 目前, 对锆石的不同颗粒 或区域获得 $\mathrm{U}-\mathrm{Pb}$ 年龄的解释主要依据以下 3 条途径: （1）锆石的显微结构及 $\mathrm{Th} / \mathrm{U}$ 值 ${ }^{[1 \sim 3]}$, (2) 锆石中的包 裹体矿物成分 ${ }^{[4,5]}$, (3) 锆石的稀土元素特征 ${ }^{[6-9]}$. 对锆 石用不同方法得到的显微结构图像能够很好地区分 岩浆锆石和变质锆石区域, 不同区域的锆石也有不 同的 Th/U 值, 但这些都不能明确给出变质锆石形成 的具体变质环境. 锆石中的矿物包裹体成分研究是 较为有效的方法, 但锆石的显微包裹体与寄主锆石 的关系较为复杂 ${ }^{[4,10]}$, 且铅石本身较小, 含包裹体的 数量较少、粒径小, 这些因素限制了该方法的使用. 锆石的稀土元素特征除与各个稀土元素进人锆石晶 格的能力大小有关外, 还和与锆石同时形成的矿物 种类有关(如石榴石、长石等), 这些矿物存在与否对 变质作用的条件(如榴辉岩相、麻粒岩相和角闪岩相 等)有重要的指示意义, 同时锆石的微区稀土元素分 析可以与微区定年相结合, 所以锆石微区的稀土元 素特征可以为锆石的形成环境提供重要的制约 ${ }^{[6-8]}$. 对高压超高压变质岩中锆石的研究目前主要是榴辉 岩相变质片麻岩 ${ }^{[7-9]}$, 对榴辉岩中铅名的稀生光素及
其他微量元素的研究还尚未见报道.

本文对大别山黄镇榴辉岩中的锆石进行了阴极 发光 (CL) 显微结构分析, 并利用激光探针等离子体 质谱(LAM-ICP-MS)对锆石的不同区域进行微量元素 测定, 结果表明榴辉岩中原岩和变质锆石具有不同 的微量元素特征, 锆石中除稀土外 $\mathrm{Nb}$, Ta 等微量元 素特征同样可以用来指示锆石的形成条件.

\section{1 样品及分析方法}

榴辉岩样品 HZ01-1-10 采自太湖县黄镇附近朱 家冲, 太湖-马庙公路南侧天然露头一个 $4 \sim 5 \mathrm{~m}$ 宽的 透镜体, 为含蓝晶石榴辉岩. 主要矿物组合为石榴石 + 绿辉石 + 角闪石 + 石英 + 钠云母 + 金红石 + 绿帘石 + 蓝晶石, 等. 这一地区的榴辉岩位于大别 超高压变质带的南部, 通常称为低温榴辉岩带, 可能 只经历了高压变质作用 ${ }^{[11,12]}$, 并且角闪岩相退变质 作用明显, 结果破坏了榴辉岩矿物之间的氧同位素 平衡 ${ }^{[13]}$. 将重约 $15 \mathrm{~kg}$ 的榴辉岩样品破碎后经摇床、 淘洗及电磁选分离出锆石, 并在双目显微镜下挑纯. 将待测的锆石颗粒置于环氧树脂样品座中, 先进行 $\mathrm{CL}$ 成像观察, 然后根据铅石不同的 CL 结构区域来 进行微量元素分析. CL 成像观察在法国 Nancy I 大 学完成. 
锆石的微量元素测定在中国科学院广州地球化 学研究所 ELAN6000 型电感耦合等离子体质谱 (ICP-MS)与 LSX-100 型激光熔蚀探针 (LAM) 的联机 上进行. 激光系统为 Nd-YAG uv 激光器, 使用波长为 $266 \mathrm{~nm}$. 样品分析时激光束直径 20 30 $\mu \mathrm{m}$, 激光熔蚀 样品的深度为 $20 \sim 40 \mu \mathrm{m}$. 工作模式为 Q-Switched 模式, 最大脉冲能量为 $3 \mathrm{~mJ} / \mathrm{pulse}$, 能量稳定性优于 $10 \%$, 具有 $1 \sim 20 \mathrm{~Hz}$ 可变脉冲频率. ICP 的有效功率为 $1000 \mathrm{~W}$, 四极杆质谱的质量分辨率为 $(0.70 \pm 0.02) \mathrm{amu}$, 偏置 电压为 $15.4 \mathrm{~V}$, 载样气体为 $\mathrm{Ar}$, 测定时用 NIST610 玻璃作为外标. 由于 $\mathrm{SiO}_{2}$ 在锆石中的含量较恒定 $(32.9 \%)$, 选择 $\mathrm{Si}$ 作为内标来消除激光能量在点分析 过程中以及分析点之间的漂移. 对于大多数元素单 点分析的相对标准偏差为 5\% 15\%, 少量浓度接近检 出限(LOD)的微量元素(如 $\mathrm{La}, \mathrm{Pr}, \mathrm{Rb}$ 等)分析的相对 标准偏差高达 20\% 40\%. 锆石微量元素的详细分析 方法已有专文介绍 ${ }^{[14,15]}$. 表 1 列出了锆石微量元素的 分析结果.

\section{2 结果及讨论}

\section{1 锆石的 CL 图像特征}

样品 HZ01-1-10 中的锆石为半自形到它形, 短柱 状到等轴状. CL 图像(图 1)显示所有分析的锆石颗粒 都有明显的核边结构, 核部的 CL 较弱为黑色, 边部 的 CL 较强呈灰白色. 这种 CL 图像的差异可能反应 了它们之间元素含量的不同, 主要是微量元素特别 是稀土元素和 $\mathrm{Th}, \mathrm{U}$ 含量的不同. 核部 CL 图像中有

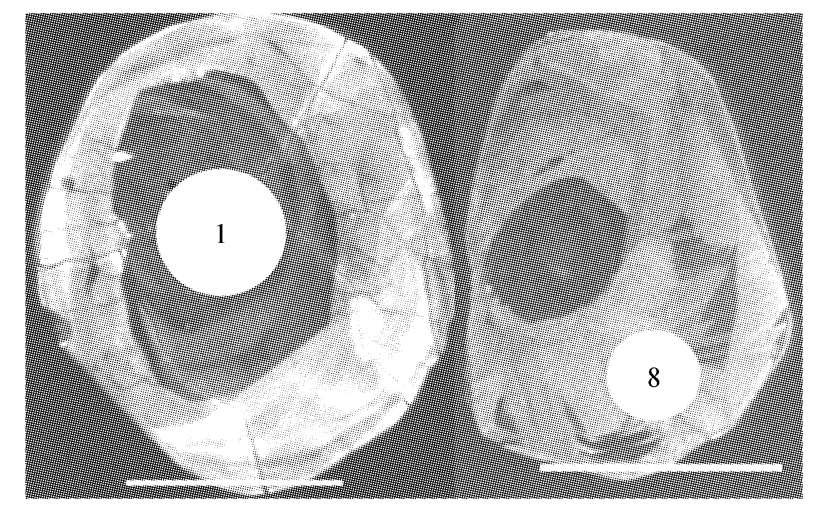

图 1 黄镇榴辉岩中锆石的 CL 图像及微量元素 分析点的位置 微标尺为 $50 \mu \mathrm{m}$
不同程度弱的环带结构(如 1,2 号颗粒), 所以核部应 为该榴辉岩样品原岩的岩浆锆石. 锆石边部较均匀, 少量有斑杂状结构, 为典型的变质锆石的特征. 由于 锆石的核边之间的界限清楚, 锆石核部的结构被边 部切割, 且少量锆石的核部有港湾状结构(如颗粒 1), 所以我们认为边部的锆石为变质增生的. 在我们的 分析点中, 1, 2, 6, 7 和 10 号分析点位于锆石的核部, 而 3, 4, 8 和 9 号分析点位于锆石的边部.

\section{2 锆石的微量元素特征}

(i ) 锆石的 Th, U 特征. 从表 1 可知, 锆石的 核部和边部 $\mathrm{Th}, \mathrm{U}$ 含量和 $\mathrm{Th} / \mathrm{U}$ 值存在较大的差异. 锆石核部有较高的 $\mathrm{Th}, \mathrm{U}$ 含量 $(\mathrm{Th}$ 为 $22.2 \sim 67.9 \mu \mathrm{g} / \mathrm{g}$; $\mathrm{U}$ 为 $427.8 \sim 1180.7 \mu \mathrm{g} / \mathrm{g}$ ), 它们的变化范围也较大, $\mathrm{Th} / \mathrm{U}$ 值为 0.04 0.05. 边部锆石的 $\mathrm{Th}(7.4 \sim 10.7 \mu \mathrm{g} / \mathrm{g})$ 、 $\mathrm{U}(333.1 \sim 453.0 \mu \mathrm{g} / \mathrm{g}$ ) 含量明显低于锆石的核部, 变化 范围较小, $\mathrm{Th} / \mathrm{U}$ 值为 $0.02 \sim 0.03$.

虽然锆石核部的 $\mathrm{Th} / \mathrm{U}$ 值较低 $(<0.1)$, 接近变质 锆石 $\mathrm{Th} / \mathrm{U}$ 值的变化范围, 但其相对于边部的变质锆 石而言偏高. 较高的 Th, U 含量和部分核部区域存在 振荡环带等特征表明其为岩浆锆石. 其 $T h, U$ 含量变 化较大的特点证明了这些元素在核部的分布有较明 显的不均一性, 与 CL 图像中存在部分环带的结果一 致. 部分基性岩中存在 $\mathrm{Th} / \mathrm{U}$ 值较低(<0.1)的岩浆锆 石已有报道 ${ }^{[16]}$, 所以本文得到黄镇榴辉岩中岩浆锆 石具有较低的 $T h / U$ 值是合理的. 而边部的变质锆石 则形成于 $T h, U$ 含量低且变化范围不大的环境.

(ii ) 稀土元素(REE). 本文分析的锆石核部和 变质边部的稀土元素具有一定的共性：所有锆石分 析点在球粒陨石标准化图中呈轻稀土亏损、重稀土逐 步富集的稀土配分模式, Ce 正异常明显, Eu 负异常强 弱不等(图 2). 这是典型的锆石稀土元素配分模式, 这种配分模式主要是由于各个稀土元素的地球化学 性质差异导致各稀土元素进人锆石晶格能力的不同 造成的 ${ }^{[17]}$.

表 1 及图 2 显示不同区域(核部和边部)锆石的稀 土元素存在明显的差异, 主要表现在以下几个方面: (1) 稀土总量: 核部岩浆锆石的稀土总量高(328 792 $\mu \mathrm{g} / \mathrm{g})$, 变化较大; 而边部变质锆石的稀土总量低, 只 有 1 145 204 $\mu \mathrm{g} / \mathrm{g}$. - (2)8重稀土富集程度：核部岩浆锆 
表 1 黄镇榴辉岩不同类型锆石微量元素组成分析结果 (Hf 为质量百分数; 其余为 $\mu \mathrm{g} / \mathrm{g}$ ) ${ }^{\mathrm{a})}$

\begin{tabular}{|c|c|c|c|c|c|c|c|c|c|}
\hline \multirow{2}{*}{ 样品 } & \multicolumn{5}{|c|}{ 岩浆锆石 } & \multicolumn{4}{|c|}{ 变质锆石 } \\
\hline & HZ-1 & HZ-2 & HZ-6 & HZ-7 & HZ-10 & HZ-3 & HZ-4 & HZ-8 & HZ-9 \\
\hline $\mathrm{Ti}$ & 389.8 & 431.2 & 205.3 & 480.6 & 550.0 & 395.7 & 378.8 & 375.3 & 714.4 \\
\hline $\mathrm{Rb}$ & bd & 0.1 & 3.2 & bd & bd & $\mathrm{bd}$ & 0.2 & 0.8 & 0.7 \\
\hline $\mathrm{Sr}$ & 4.7 & 1.6 & 0.8 & 1.3 & 0.5 & 1.3 & 3.0 & 0.1 & 2.5 \\
\hline $\mathrm{Y}$ & 426.5 & 877.5 & 597.8 & 696.1 & 365.6 & 298.1 & 249.0 & 385.5 & 387.9 \\
\hline $\mathrm{Nb}$ & 6.1 & 19.7 & 12.5 & 9.5 & 3.8 & 0.5 & 1.4 & 1.0 & 0.9 \\
\hline $\mathrm{La}$ & 0.6 & 0.4 & 2.8 & bd & 2.8 & 2.3 & 0.7 & 0.3 & 0.4 \\
\hline $\mathrm{Ce}$ & 15.5 & 26.2 & 31.6 & 19.0 & 14.4 & 7.4 & 6.4 & 4.6 & 7.1 \\
\hline $\operatorname{Pr}$ & 1.2 & bd & 1.8 & bd & 0.2 & 0.2 & 0.2 & 0.1 & $\mathrm{~d}$ \\
\hline $\mathrm{Nd}$ & 4.1 & 0.0 & 11.0 & 1.0 & 3.0 & 0.3 & 0.5 & 0.8 & 0.9 \\
\hline $\mathrm{Sm}$ & bd & 1.5 & 8.5 & 0.8 & 2.5 & 1.6 & 2.1 & bd & 1.6 \\
\hline $\mathrm{Eu}$ & 0.8 & 0.8 & 2.2 & 0.5 & 0.9 & 0.6 & 0.4 & 0.7 & 0.4 \\
\hline $\mathrm{Gd}$ & 2.0 & 8.4 & 16.5 & 10.4 & 15.5 & 5.4 & 7.0 & 8.3 & 8.7 \\
\hline $\mathrm{Tb}$ & 2.3 & 2.8 & 5.7 & 3.7 & 3.3 & 3.4 & 2.2 & 3.1 & 2.4 \\
\hline Dy & 26.4 & 52.8 & 53.1 & 43.7 & 35.3 & 22.7 & 28.2 & 40.4 & 32.8 \\
\hline Ho & 14.6 & 21.3 & 17.4 & 19.0 & 12.9 & 8.0 & 8.1 & 12.3 & 11.4 \\
\hline $\mathrm{Er}$ & 70.1 & 151.6 & 65.1 & 117.6 & 53.1 & 26.6 & 30.8 & 42.8 & 34.3 \\
\hline $\mathrm{Tm}$ & 21.0 & 37.2 & 15.9 & 27.6 & 13.2 & 5.9 & 4.7 & 8.3 & 6.1 \\
\hline $\mathrm{Yb}$ & 173.6 & 414.9 & 157.6 & 332.9 & 145.1 & 52.7 & 49.6 & 72.4 & 63.1 \\
\hline $\mathrm{Lu}$ & 30.5 & 74.3 & 29.7 & 61.5 & 26.1 & 7.7 & 7.7 & 9.8 & 6.8 \\
\hline $\mathrm{Hf}$ & 0.56 & 1.22 & 1.84 & 1.44 & 1.27 & 1.14 & 1.05 & 1.10 & 1.31 \\
\hline $\mathrm{Ta}$ & 2.9 & 12.1 & 2.7 & 5.5 & 3.9 & 1.5 & 1.2 & 1.1 & 0.7 \\
\hline $\mathrm{Pb}$ & 40.3 & 363.7 & 77.4 & 85.7 & 32.4 & 26.6 & 19.5 & 18.9 & 30.9 \\
\hline Th & 22.2 & 67.9 & 46.1 & 61.5 & 29.1 & 8.8 & 7.4 & 10.7 & 10.0 \\
\hline $\mathrm{U}$ & 427.8 & 1077.1 & 974.2 & 1180.7 & 737.2 & 376.3 & 333.1 & 423.0 & 453.0 \\
\hline $\mathrm{Nb} / \mathrm{Ta}$ & 2.1 & 1.6 & 4.6 & 1.7 & 1.0 & 0.3 & 1.2 & 0.9 & 1.3 \\
\hline $\mathrm{Th} / \mathrm{U}$ & 0.05 & 0.06 & 0.05 & 0.05 & 0.04 & 0.02 & 0.02 & 0.03 & 0.02 \\
\hline$\Sigma$ REE & 362.8 & 792.1 & 418.9 & 637.6 & 328.2 & 144.6 & 148.6 & 204.0 & 175.9 \\
\hline$\Sigma$ HREE & 340.6 & 763.2 & 361.0 & 616.4 & 304.5 & 132.2 & 138.5 & 197.6 & 158.7 \\
\hline$(\mathrm{Yb} / \mathrm{Gd})_{\mathrm{CN}}$ & 107.4 & 59.6 & 11.5 & 38.9 & 11.3 & 11.9 & 8.6 & 10.5 & 8.8 \\
\hline
\end{tabular}

a) bd 为低于检出限

石的重稀土 $(\mathrm{Gd} \sim \mathrm{Lu})$ 元素含量高(304.5 763.2 $\mu \mathrm{g} / \mathrm{g})$, 重稀土的分异程度较大 $\left((\mathrm{Yb} / \mathrm{Gd})_{\mathrm{CN}}=11.3 \sim 107.4\right)$; 边 部变质锆石的重稀土元素含量低(132.2 197.6 $\mu \mathrm{g} / \mathrm{g})$, 重稀土的分异程度小 $\left((\mathrm{Yb} / \mathrm{Gd})_{\mathrm{CN}}=8.6 \sim 11.9\right)$. 边部锆 石这种类型的稀土配分模式是典型的与石榴石平衡 的锆石的特征, 由于石榴石中大量富集重稀土导致 与之同时形成的锆石重稀土含量降低 ${ }^{[7,8]}$. 这说明该 样品锆石的变质边部形成于榴辉岩相变质阶段, 对 其进行微区定年可以准确确定榴辉岩相变质作用的 年龄. (3) $\mathrm{Ce}$ 和 $\mathrm{Eu}$ 异常: 核部和边部锆石的正 $\mathrm{Ce}$ 异 常较为接近, 而 $\mathrm{Eu}$ 负异常有很大程度的不同(图 2). 核部的 $\mathrm{Eu}$ 异常不明显, 而边部锆石具有清楚的 $\mathrm{Eu}$ 负异常. Rubatto 等人 ${ }^{[7]}$ 认为榴辉岩相锆石与原岩岩
浆锆石相比具有更小的 $E u$ 异常, 但 Hermann 等人 ${ }^{[8]}$ 对哈萨克斯坦 Kokchetav 超高压变质岩中不同锆石 的详细研究后, 认为锆石 $\mathrm{Eu}$ 异常与全岩存在 $\mathrm{Eu}$ 负异 常与否有关. 我们的样品中部分榴辉岩相变质锆石 的 $\mathrm{Eu}$ 异常比原岩锆石明显, 这说明锆石的 $\mathrm{Eu}$ 异常可 能不是判定榴辉岩相变质锆石的有效指标.

Hoskin 等人 ${ }^{[18]}$ 对部分变质锆石的稀土元素进行 研究后认为, 变质过程对锆石的 REE 分配影响不明 显. Schaltegger 等人 ${ }^{[9]}$ 对含石榴石麻粒岩样品的研究 结果同样支持这一结论. 由于 Hoskin 等人 ${ }^{[18]}$ 样品的 地质特征没有详细描述, 我们不能肯定他们的样品 中是否存在石榴石这一变质矿物. 而 Schaltegger 等 人部研究的样品中存在明显的部分熔融现象, 部分变 


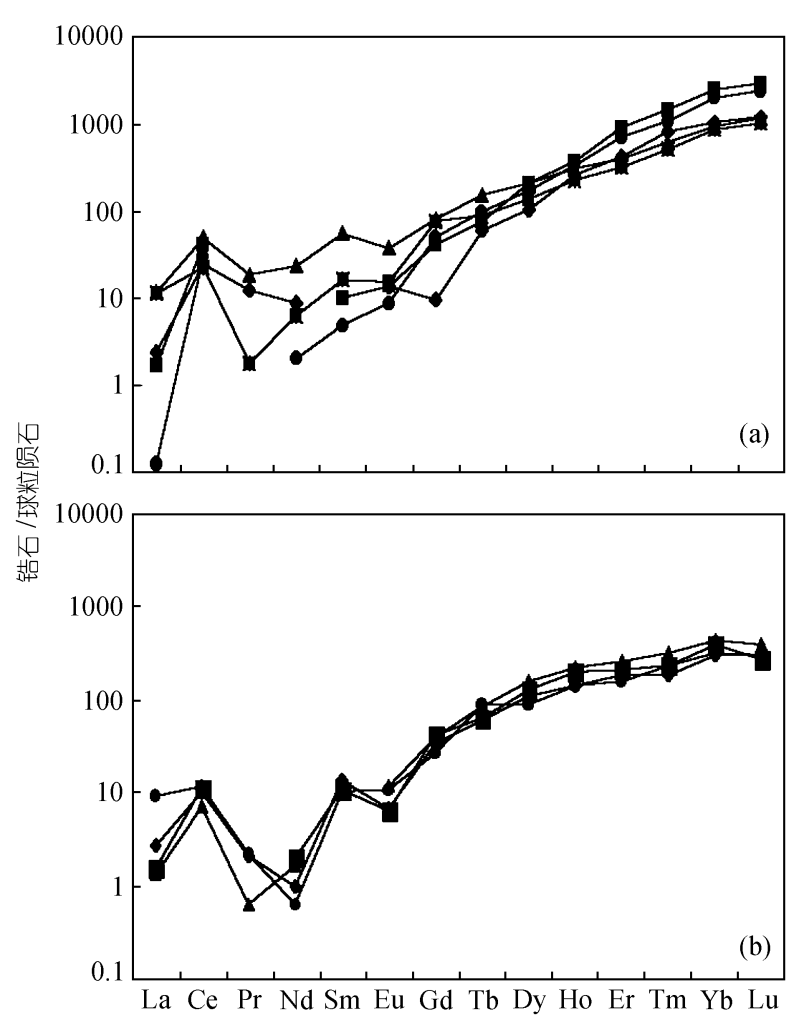

图 2 黄镇榴辉岩不同区域锆石球粒陨石标准化的 REE 配分模式

(a) 原岩中的岩浆钻石; (b) 变质增生铅石

质锆石是从融体中结晶出来的, 结果表现出岩浆锆 石的稀土元素特征, 因此不能保证这些新生的变质 锆石是与石榴石平衡的. 他们的样品中同样含有重 稀土亏损的变质锆石, 这些锆石可能是与石榴石平 衡结晶的. 本文结果及其他高压超高压变质条件下 形成的变质锆石稀土元素的研究结果 ${ }^{[6-8]}$ 表明, 高压 超高压变质岩中与石榴石平衡生长的变质铅石具有 稀土总量低及重稀土相对亏损的特征.

(iii) 其他微量元素. 不同区域锆石的大离子亲 石元素(LILE)含量都非常低, Rb, Sr 含量不超过 5.0 $\mu \mathrm{g} / \mathrm{g}$ ，与 $\mathrm{Li}$ 等人 ${ }^{[14]}$ 对不同来源的铅石进行微量元素 测定的结果类似. 表明这类微量元素不易进入锆石 的晶格中.

锆石的核部和边部 $\mathrm{Nb}, \mathrm{Ta}$ 含量差别明显(图 3), 核部 $\mathrm{Nb}$, Ta 含量较高, 分别为 3.8 19.7 和 2.7 12.1 $\mu \mathrm{g} / \mathrm{g}, \mathrm{Nb} / \mathrm{Ta}$ 值也偏大, 为 $1.0 \sim 4.6$. 而边部的 $\mathrm{Nb}, \mathrm{Ta}$ 含量和 $\mathrm{Nb} / \mathrm{Ta}$ 值分别为 $0.5 \sim 1.4 \mu \mathrm{g} / \mathrm{g}, 0.7 \sim 1.5 \mu \mathrm{g} / \mathrm{g}$ 和

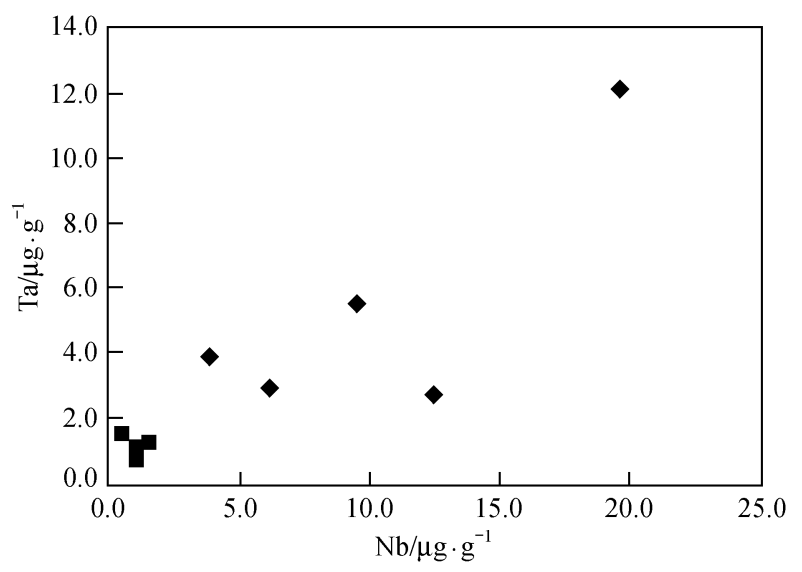

图 3 锆石的 Nb-Ta 图解 正方形为变质钻石, 菱形为岩浆钻石

0.3 1.3. 锆石中的低 $\mathrm{Nb} / \mathrm{Ta}$ 值可能是由于 $\mathrm{Ta}$ 更易进 人锆石晶格 ${ }^{[14]}$. 变质边部锆石 $\mathrm{Nb}, \mathrm{Ta}$ 含量和 $\mathrm{Nb} / \mathrm{Ta}$ 值低于核部的岩浆锆石, 表明在边部变质铅石形成 的同时有富集 $\mathrm{Nb}, \mathrm{Ta}$ 和具较高 $\mathrm{Nb} / \mathrm{Ta}$ 值的矿物形成. 金红石是榴辉岩中常见的高压超高压变质矿物, 它 强烈富集高场强元素, 特别是 $\mathrm{Nb}$ 和 $\mathrm{Ta}$, 并且有较高 的 $\mathrm{Nb} / \mathrm{Ta}$ 值 ${ }^{[19]}$. 这一关系表明, 边部的变质锆石 $\mathrm{Nb}$, $\mathrm{Ta}$ 特征是与金红石平衡结晶的结果. 由于金红石是 榴辉岩中的峰期变质矿物, 所以该样品中锆石边部 的变质部分应该是形成于榴辉岩相变质作用期间. 离子探针定年结果同样显示, 该样品的锆石的边部 形成于印支期(235 Ma), 核部岩浆锆石形成于早元古 代(约 $1900 \mathrm{Ma}$ )(陈道公等, 未发表资料).

\section{3 结论}

(i) 铅石的 CL 显微图像结果显示, 黄镇榴辉岩 的锆石存在明显的核边结构, 核部为原岩的岩浆锆 石, 边部为变质锆石.

(ii) 除锆石的稀土元素外, 锆石的 Nb, Ta 含量 和 $\mathrm{Nb} / \mathrm{Ta}$ 值同样可以用来制约锆石的形成条件. 锆石 的稀土元素配分模式和 $\mathrm{Nb}$, Ta 等微量元素特征表明, 边部的变质锆石形成于榴辉岩相变质作用期间, 核 部为原岩中的岩浆锆石.

(iii) 锆石的微量元素分析表明, 榴辉岩相变质 环境中微量元素含量较低, 因为榴辉岩相变质作用 形成锆石的微量元素含量较低(如 Th, U, REE, Nb 和 $\mathrm{Ta}$ 等)。 
致谢 郑永飞教授审阅全文并提出宝贵修改意见，野外工 作得到范良红工程师的帮助, 样品分析期间李献华研究员 和梁细荣研究员给予了一定的指导和帮助, 广州地区科学 仪器协作共用网提供部分资助。作者在此一并致谢. 本工 作受国家重点基础研究发展规划项目(G1999075503)和国 家自然科学基金(批准号: 40033010, 49973019)的资助.

\section{参考文献}

1 Rubatto D, Gebauer D, Fanning M. Jurassic formation and Eocene subductions for the geodynamic evolution of the Central and Western Alps. Contrib Mineral Petrol, 1998, 132: 269 287

2 Rubatto D, Gebauer G, Compagnoni R. Dating of eclogite-facies zircons: The age of Alpine metamorphism in the Sesia-Lanzo Zone (Western Alps). Earth and Planetary Science Letters, 1999, 167: $141 \sim 158$

3 Vavra G, Gebauer D, Schmid R, et al. Multiple zircon growth and recrystallization during polyphase Late Carboniferous to Triassic metamorphism in granulites of the Ivrea Zone (Southern Alps): An ion microprobe (SHRIMP) study. Contrib Mineral Petrol, 1996, 122: $337 \sim 358$ pressure metamorphism and evidence for very rapid exhumition in Dora Maria Massif, Western Alps. Lithos, 1997, 41: 5 24

5 Katayama I, Maruyama S, Parkinson C D, et al. Ion micro-probe $\mathrm{U}-\mathrm{Pb}$ zircon geochronology of peak and retrograde stages of ultrahigh-pressure metamorphic rocks from the Kokchetav massif, northern Kazakhstan. Earth and Planetary Science Letters, 2001, 188: $185 \sim 198$

6 Rowley D B, Xue F, Tucker R D, et al. Ages of ultra high pressure metamorphism and protolith orthgneisses from the eastern Dabie Shan: U/Pb zircon geochronology. Earth Planet Science Letters, 1997, 151: 191 20

7 Rubatto D, Williams I S. Imageing, trace element geochemistry and mineral inclusions: Linking $\mathrm{U}-\mathrm{Pb}$ ages with metamorphic conditions. EOS, 2000, 21: 25

8 Hermann J, Rubatto D, Korsakov A. Multiple zircon growth during fast exhumation of diamondiferous, deeply subducted continental crust (Kokchetav Massif, Kazakhstan). Contrib Mineral Petrol, 2001, 141:
9 Schaltegger U, Fanning C M, Gunther D, et al. Growth, annealing and recrystllization of zircon and preservation of monazite in high-grade metamorphism: Conventional and in-situ U-Pb isotope, cathodoluminescence and microchemical evidence. Contrib Mineral Petrol, 1999, 134: 186 201

10 Liu J, Ye K, Maruyama S, et al. Mineral inclusions in zircon from gneisses in the Ultrahigh-pressure zone of the Dabie Mountains, China. Journal of Geology, 2001, 109: 523 535

11 Carswell D, O'Brien P, Wilson R, et al. Thermobarometry of phengite-bearing eclogites in the Dabie mountains of central China. J Metamorphic Geol, 1997, 15: 239 252

12 Okay A. Petrology of a diamond and coesite-bearing metamorphic terrain: Dabie Shan, China. Eur J Mineral, 1993, 5: 659 675

13 郑永飞, 付斌, 李一良, 等. 大别山超高压变质作用期前和期后 水-岩相互作用的氢氧同位素证据. 中国科学, D辑, 1999, 29(2): $113 \sim 121$

14 Li X, Liang X, Sun M, et al. Geochronology and geochemistry of single-grain zircons: Simultaneous in-situ analysis of U-Pb age and trace elements by LAM-ICP-MS. Eur J Mineral, 2000, 12: $1015 \sim 1024$

15 梁细荣, 李献华, 刘永康, 等. 激光探针等离子体质谱同时测定锆 石微区铀-铅年龄及微量元素. 岩矿测试, 1999, 18(4): 253 258

16 Gebauer D. A P-T-t Path for an (Ultra?) High-Pressure ultramafic/mafic rock-association and its felsic country-rocks based on SHRIMP-dating of magmatic and metamorphics zircon domains. Example: Alps Arami Central Swiss Alps. In: Reading the Isotopic Code, Geophsical Monograph 95. Washington D C: Am Geophy Union, 1996. 307 329

17 Hinton R W, Upton B G J. The chemistry of zircon: Variations within and between large crystals from syenite and alkali basalt xenoliths. Geochim Cosmochim Acta, 1991, 55(11): 3287 3302

18 Hoskin $\mathrm{P}$ W O, Ireland $\mathrm{T}$ R. Rare earth element chemistry of zircon and its use as a provenance indicator. Geology, 2000, 28(7): $627 \sim 630$

19 Rudnick R, Barth M, Horn I, et al. Rutile-bearing refractory eclogites: Missing link between continents and depleted mantle. Science, 2000, 287: 278 281 